\title{
On the existence of self-similar spherically symmetric wave maps coupled to gravity
}

\author{
Piotr Bizon ${ }^{1}$ and Arthur Wasserman ${ }^{2}$ \\ ${ }^{1}$ Institute of Physics, Jagellonian University, Kraków, Poland \\ 2 Department of Mathematics, University of Michigan, Ann Arbor, MI, USA
}

Received 15 February 2002

Published 5 June 2002

Online at stacks.iop.org/CQG/19/3309

\begin{abstract}
We present a detailed analytical study of spherically symmetric self-similar solutions in the $S U(2)$ sigma model coupled to gravity. Using a shooting argument, we prove that there is a countable family of solutions which are analytic inside the past self-similarity horizon. In addition, we show that for sufficiently small values of the coupling constant these solutions possess a regular future self-similarity horizon and thus are examples of naked singularities. One of the solutions constructed here has been recently found as the critical solution at the threshold of black-hole formation.
\end{abstract}

PACS numbers: 0462,0465

\section{Introduction}

In this paper, we continue our investigations, started in [1] (referred to as I), of wave maps coupled to gravity, that is, solutions of Einstein's equations with an $S U(2)$ sigma field as matter. We found numerically in I that for $\alpha<1 / 2$ ( $\alpha$ is the dimensionless coupling constant) the model admits a countable family of continuously self-similar (CSS) solutions, labelled by an integer nodal index $n=0,1, \ldots$, that are analytic inside the past light cone of the singularity. We also provided evidence that the $n$th CSS solution can be extended to the future light cone of the singularity if $\alpha<\alpha_{n}$, where $\left\{\alpha_{n}\right\}$ is an increasing sequence bounded above by $1 / 2$. The purpose of this paper is to make the results of I (except for the ordering of $\alpha_{n}$ ) into theorem-proof rigorous mathematics. This is accomplished by applying a shooting argument to the resulting dynamical system. We note that the case $\alpha=0$ was previously analysed in [2].

The physical importance of the CSS solutions considered here was discussed in I. In particular, we conjectured that in a certain parameter range $\left(\alpha_{0}<\alpha<\alpha_{1}\right)$ the $n=1$ solution is a critical solution at the threshold of black-hole formation. This conjecture has been recently confirmed in numerical studies of the critical behaviour [3] and in the linear stability analysis [4]. As far as we know, this is the only case where the existence of a self-similar solution, 
which was numerically found as the critical solution in gravitational collapse, has been established rigorously.

\section{Setup}

For the reader's convenience, we repeat from I the basic setting for the problem. Let $X: M \rightarrow N$ be a map from a spacetime $\left(M, g_{a b}\right)$ into a Riemannian manifold $\left(N, G_{A B}\right)$. Wave maps coupled to gravity are defined as extrema of the action

$$
S=\int_{M}\left(\frac{R}{16 \pi G}+L_{W M}\right) \mathrm{d} v_{g}
$$

with the Lagrangian density

$$
L_{W M}=-\frac{f_{\pi}^{2}}{2} g^{a b} \partial_{a} X^{A} \partial_{b} X^{B} G_{A B} .
$$

Here $G$ is Newton's constant and $f_{\pi}^{2}$ is the wave map coupling constant. The product $\alpha=4 \pi G f_{\pi}^{2}$ is dimensionless. The field equations derived from (1) are the wave map equation

$$
\square_{g} X^{A}+\Gamma_{B C}^{A}(X) \partial_{a} X^{B} \partial_{b} X^{C} g^{a b}=0,
$$

where $\Gamma_{B C}^{A}(X)$ are the Christoffel symbols of the target metric $G_{A B}$ and $\square_{g}$ is the d'Alembertian associated with the metric $g_{a b}$, and the Einstein equations $R_{a b}-\frac{1}{2} g_{a b} R=$ $8 \pi G T_{a b}$ with the stress-energy tensor

$$
T_{a b}=f_{\pi}^{2}\left(\partial_{a} X^{A} \partial_{b} X^{B}-\frac{1}{2} g_{a b}\left(g^{c d} \partial_{c} X^{A} \partial_{d} X^{B}\right)\right) G_{A B} .
$$

As a target manifold, we take the 3 -sphere $S^{3}$ with the standard metric in polar coordinates $X^{A}=(F, \Theta, \Phi)$,

$$
G_{A B} \mathrm{~d} X^{A} \mathrm{~d} X^{B}=\mathrm{d} F^{2}+\sin ^{2} F\left(\mathrm{~d} \Theta^{2}+\sin ^{2} \Theta \mathrm{d} \Phi^{2}\right) .
$$

For the domain manifold, we assume spherical symmetry and use Schwarzschild coordinates

$$
g_{a b} \mathrm{~d} x^{a} \mathrm{~d} x^{b}=-e^{-2 \delta} A \mathrm{~d} t^{2}+A^{-1} \mathrm{~d} r^{2}+r^{2}\left(\mathrm{~d} \theta^{2}+\sin ^{2} \theta \mathrm{d} \phi^{2}\right),
$$

where $\delta$ and $A$ are functions of $(t, r)$. Next, we assume that the wave maps are corotational, that is,

$$
F=F(t, r), \quad \Theta=\theta, \quad \Phi=\phi .
$$

Equation (3) reduces then to the single semilinear wave equation

$$
\square_{g} F-\frac{\sin (2 F)}{r^{2}}=0,
$$

where

$$
\square_{g}=-e^{\delta} \partial_{t}\left(e^{\delta} A^{-1} \partial_{t}\right)+\frac{e^{\delta}}{r^{2}} \partial_{r}\left(r^{2} e^{-\delta} A \partial_{r}\right),
$$

and the Einstein equations become

$$
\begin{aligned}
& \partial_{t} A=-2 \alpha r A\left(\partial_{t} F\right)\left(\partial_{r} F\right), \\
& \partial_{r} \delta=-\alpha r\left(\left(\partial_{r} F\right)^{2}+A^{-2} e^{2 \delta}\left(\partial_{t} F\right)^{2}\right), \\
& \partial_{r} A=\frac{1-A}{r}-\alpha r\left(A\left(\partial_{r} F\right)^{2}+A^{-1} e^{2 \delta}\left(\partial_{t} F\right)^{2}+2 \frac{\sin ^{2} F}{r^{2}}\right) .
\end{aligned}
$$

These equations are invariant under dilations $(t, r) \rightarrow(\lambda t, \lambda r)$, so it is natural to look for continuously self-similar (CSS) solutions, that is solutions which are left invariant by the 
action of the homothetic Killing vector $K=t \partial_{t}+r \partial_{r}$. To study such solutions, it is convenient to use similarity variables $\rho=r /(-t)$ and $\tau=-\ln (-t)$. Then $K=-\partial_{\tau}$, so CSS solutions do not depend on $\tau$. Assuming this and using an auxiliary function $Z=e^{\delta} \rho / A$, we reduce equations (8)-(12) to the system of ordinary differential equations (where prime is $\mathrm{d} / \mathrm{d} \rho$ ):

$$
\begin{aligned}
& F^{\prime \prime}+\frac{2}{\rho} F^{\prime}-\alpha\left(1+Z^{2}\right) \rho F^{\prime 3}-\frac{\sin (2 F)}{A \rho^{2}\left(1-Z^{2}\right)}=0, \\
& A^{\prime}=-2 \alpha \rho A F^{\prime 2}, \\
& \rho Z^{\prime}=Z\left(1+\alpha\left(1-Z^{2}\right) \rho^{2}{F^{\prime}}^{2}\right), \\
& \rho A^{\prime}=1-A-\alpha\left(\rho^{2} A\left(1+Z^{2}\right) F^{\prime 2}+2 \sin ^{2} F\right) .
\end{aligned}
$$

The combination of (14) and (16) yields the constraint

$$
1-A-2 \alpha \sin ^{2} F+\alpha A \rho^{2} F^{\prime 2}\left(1-Z^{2}\right)=0 .
$$

This system of equations has a fixed singularity at the centre $\rho=0$ and moving singularities at points where $Z(\rho)= \pm 1$ and/or $A(\rho)=0$. In terms of the similarity coordinate $\rho$, the metric (6) takes the form

$\mathrm{d} s^{2}=A^{-1}\left(1-Z^{-2}\right) \rho^{2} \mathrm{~d} t^{2}+2 A^{-1} t \rho \mathrm{d} t \mathrm{~d} \rho+A^{-1} t^{2} \mathrm{~d} \rho^{2}+t^{2} \rho^{2}\left(\mathrm{~d} \theta^{2}+\sin ^{2} \theta \mathrm{d} \phi^{2}\right)$,

hence the hypersurfaces $Z= \pm 1$ are null (provided that $A>0$ ). The first $\rho_{1}$ where $Z\left(\rho_{1}\right)=1$ is the locus of the past light cone of the singularity at the origin $(t=0, r=0)$ (in what follows, we shall refer to the past and future light cones of the singularity as the past and future self-similarity horizons (SSH)). By rescaling, $\rho \rightarrow \rho / \rho_{1}$, one can always locate the past self-similarity horizon at $\rho_{1}=1$, that is $Z(1)=1$. To ensure regularity of solutions in the interval $0 \leqslant \rho \leqslant 1$, equations (13)-(17) must be supplemented by the boundary conditions at both endpoints,

$$
\begin{aligned}
& F(0)=0, \quad F^{\prime}(0)=c, \quad Z(0)=0, \quad A(0)=1, \\
& F(1)=\frac{\pi}{2}, \quad F^{\prime}(1)=b, \quad Z(1)=1, \quad A(1)=1-2 \alpha,
\end{aligned}
$$

where $c$ and $b$ are free parameters. At this point, it might not be obvious why the boundary condition $F(1)=\pi / 2$ in (20) needs to be chosen, as one could naively think of any solution of $\sin (2 F(1))=0$. We shall show below that $F(1)=\pi / 2$ is the only possibility.

Our main result is the following theorem:

Theorem 1. For any $0 \leqslant \alpha<1 / 2$ and any non-negative integer $n$, equations (13)-(17) have an analytic solution $\left(F_{n}, A_{n}, Z_{n}\right)$ which satisfies the boundary conditions (19)-(20) and has precisely $n$ oscillations of $F_{n}(\rho)$ around $\pi / 2$.

In the next section, we shall prove this theorem using a shooting technique. The numerical evidence for theorem 1 was given in I. The case $\alpha=0$ was proved previously in [2], so hereafter we assume that $0<\alpha<1 / 2$.

\section{Proof of theorem 1}

For convenience, we rewrite equations (13)-(15) in terms of $H=F-\pi / 2$ :

$$
H^{\prime \prime}+\frac{2}{\rho} H^{\prime}-\alpha\left(1+Z^{2}\right) \rho H^{\prime 3}+\frac{\sin (2 H)}{A \rho^{2}\left(1-Z^{2}\right)}=0
$$




$$
\begin{aligned}
& A^{\prime}=-2 \alpha \rho A H^{\prime 2}, \\
& \rho Z^{\prime}=Z\left(1+\alpha\left(1-Z^{2}\right) \rho^{2} H^{\prime 2}\right) .
\end{aligned}
$$

The constraint becomes

$$
1-2 \alpha-A+2 \alpha \sin ^{2} H+\alpha A \rho^{2} H^{\prime 2}\left(1-Z^{2}\right)=0 .
$$

The initial conditions at $\rho=0$ are

$$
H(0)=-\frac{\pi}{2}, \quad H^{\prime}(0)=c, \quad A(0)=1, \quad Z(0)=0, \quad Z^{\prime}(0)=1 .
$$

Note that the above equations have a residual scaling symmetry $\rho \rightarrow \lambda \rho$. The initial condition $Z^{\prime}(0)=1$ is imposed temporarily in order to fix the scale. We shall refer to solutions of equations (21)-(24) satisfying the initial conditions (25) as $c$-orbits. In the appendix, we show that $c$-orbits exist locally and are analytic in $\rho$ and $c$. Now we shall show that $c$-orbits can be extended up to a point $\rho_{1}$ at which $Z\left(\rho_{1}\right)=1$.

Proposition 2. For any $0<\alpha<1 / 2$ and $c>0$ there is a $\rho_{1}(c) \in(\sqrt{1-2 \alpha}, 1)$, such that the c-orbit is defined for all $\rho<\rho_{1}$ and $\lim _{\rho \rightarrow \rho_{1}} Z(\rho)=1$. Furthermore, the following limits exist:

$$
\begin{gathered}
-\frac{\pi}{2}<\bar{H} \stackrel{\text { def }}{=} \lim _{\rho \rightarrow \rho_{1}} H(\rho)<\frac{\pi}{2}, \quad \bar{A} \stackrel{\text { def }}{=} \lim _{\rho \rightarrow \rho_{1}} A(\rho)=1-2 \alpha \cos ^{2} \bar{H}, \\
\lim _{\rho \rightarrow \rho_{1}}\left(1-Z^{2}\right) H^{\prime 2}=0 .
\end{gathered}
$$

Proof. Let the maximum domain of definition of the $c$-orbit be $0 \leqslant \rho<\rho_{1}$ and assume that $Z(\rho)<1$ in this interval. Then, from constraint (24) we have $A \geqslant 1-2 \alpha>0$ and hence $\bar{A}=\lim _{\rho \rightarrow \rho_{1}} A(\rho)>0$ ( $\bar{A}$ exists since $A(\rho)$ is monotone decreasing). By (23) $Z^{\prime} \geqslant 0$, hence $\bar{Z}=\lim _{\rho \rightarrow \rho_{1}} Z(\rho)$ exists. If $\bar{Z}<1$, then from constraint (24) $H^{\prime 2}$ is bounded so $\bar{H}=\lim _{\rho \rightarrow \rho_{1}} H(\rho)$ exists, which in turn implies, again by (24), that $\lim _{\rho \rightarrow \rho_{1}} H^{\prime}$ exists. Thus, $H, H^{\prime}, A$ and $Z$ all have finite limits at $\rho_{1}$ and therefore the $c$-orbit may be continued beyond $\rho_{1}$ contradicting the maximality of $\rho_{1}$. We conclude that $\bar{Z}=1$.

Now, we must show that $\bar{H} \in(-\pi / 2, \pi / 2)$ exists. Since $\bar{Z}=1$, we may no longer conclude that $H^{\prime 2}$ is bounded but from equation $(22)$ we get $(\ln A)^{\prime}=-2 \alpha \rho H^{\prime 2}$, so $H^{\prime 2}$ is integrable near $\rho_{1}$ which implies that $H^{\prime}$ is absolutely integrable $\left(\left|H^{\prime}\right|<1+H^{\prime 2}\right)$ and thus $\bar{H}$ exists. From constraint (24), $H(\rho)= \pm \pi / 2$ for some $0<\rho<\rho_{1}$ is not possible since $1-A>0$. Thus, $-\pi / 2<H(\rho)<\pi / 2$ and so $-\pi / 2 \leqslant \bar{H} \leqslant \pi / 2$. In fact, for $\rho \geqslant \rho_{1} / 2$ we have $1-A \geqslant \sigma>0$, so $2 \alpha \cos ^{2} H \geqslant \sigma>0$ (remember that we assume $\alpha>0$ ), hence $H$ is uniformly bounded away from $\pm \pi / 2$, and thus $-\pi / 2<\bar{H}<\pi / 2$.

To prove $\bar{A}=1-2 \alpha \cos ^{2} \bar{H}$, note that by (24) $d=\lim _{\rho \rightarrow \rho_{1}} H^{\prime 2}\left(1-Z^{2}\right)$ exists and is finite. Hence, by (23) $\lim _{\rho \rightarrow \rho_{1}} Z^{\prime}$ exists and is finite, so $1-Z^{2}=O\left(\rho-\rho_{1}\right)$ near $\rho_{1}$. If $d \neq 0$, then $H^{\prime 2}(\rho) \sim d /\left(\rho_{1}-\rho\right)$ would not be integrable near $\rho_{1}$, thus $d$ must be zero. Inserting this into (24) we get $\bar{A}=1-2 \alpha \cos ^{2} \bar{H}$.

Next, $(Z / \rho)^{\prime}>0$ by (23) and $\lim _{\rho \rightarrow 0}(Z / \rho)=1$ by L'Hôpital's rule, hence $Z>\rho$ for all $\rho>0$, and thus $\rho_{1}<1$. Finally, from (22) and (23)

$$
\left(\frac{A Z^{2}}{\rho^{2}}\right)^{\prime}=-\frac{2 Z^{4} A \alpha H^{\prime 2}}{\rho}<0,
$$

and since $\lim _{\rho \rightarrow 0}\left(A Z^{2} / \rho^{2}\right)=1$, we have $\left(A Z^{2} / \rho^{2}\right) \leqslant 1$ and hence $\rho_{1}>\sqrt{A} \geqslant \sqrt{1-2 \alpha}$.

If $Z\left(\rho_{2}\right)=1$ for some $\rho_{2}<\rho_{1}$, we replace $\rho_{1}$ by $\rho_{2}$ in the above arguments.

Corollary 3. The function $\rho_{1}(c)$ is continuous. 
Proof. Let $\tilde{c}$ be given and let $\epsilon>0$. By proposition 2, $\rho_{1}(\tilde{c})$ is defined. The function $Z(\rho)$ is monotone increasing for $\rho<\rho_{1}(\tilde{c})$, so $Z\left(\rho_{1}(\tilde{c})-\epsilon, \tilde{c}\right)<1$, hence for all $c$ sufficiently close to $\tilde{c}, Z\left(\rho_{1}(\tilde{c})-\epsilon, c\right)<1$, and thus $\rho_{1}(c)>\rho_{1}(\tilde{c})-\epsilon$. To show that $\rho_{1}(c)<\rho_{1}(\tilde{c})+\epsilon$ for all $c$ sufficiently close to $\tilde{c}$, we assume otherwise and get a contradiction. By the mean-value theorem $Z\left(\rho_{1}(\tilde{c})+\epsilon, c\right)-Z(\rho, c)=Z^{\prime}(\xi, c)\left(\rho_{1}(\tilde{c})+\epsilon-\rho\right)$. By continuity $Z(\rho, c)$ is close to $Z(\rho, \tilde{c})$ and $Z(\rho, \tilde{c})$ is close to 1 if $\rho$ is close to $\rho_{1}(\tilde{c})$, hence $Z(\rho, c)$ is arbitrarily close to 1. However, $Z^{\prime}(\rho, c)>Z(\rho, c) / \rho>1$, so $Z\left(\rho_{1}(\tilde{c})+\epsilon, c\right)>Z(\rho, c)+\epsilon>1$, which is a contradiction. Thus, $\rho_{1}(c)<\rho_{1}(\tilde{c})+\epsilon$.

Lemma 4. $H^{\prime}(\rho)$ is bounded near $\rho_{1}$ if and only if $\bar{H}=0$.

Proof. Suppose that $\bar{H} \neq 0$ and $H^{\prime}(\rho)$ is bounded. Then, in (21) we have

$$
H^{\prime \prime}=\text { bounded terms }-\frac{\sin 2 H}{A \rho^{2}\left(1-Z^{2}\right)} \sim \frac{b}{\rho_{1}-\rho},
$$

where $b \neq 0$. This contradicts that $H^{\prime}(\rho)$ is bounded near $\rho_{1}$ and concludes the 'only if' part of lemma 4.

Suppose now that $H\left(\rho_{1}\right)=0$ and $H^{\prime}(\rho)$ is unbounded. Without the loss of generality, we consider the case that $H(\rho)<0$ and $H^{\prime}(\rho)>0$ near $\rho_{1}$. Dividing equation $(21)$ by $H^{\prime}$ and integrating from $\rho$ to $\rho_{1}$, we obtain

$$
\int_{\rho}^{\rho_{1}}\left(\frac{H^{\prime \prime}}{H^{\prime}}+\frac{2}{\rho}-\alpha\left(1+Z^{2}\right) \rho H^{\prime 2}+\frac{\sin (2 H)}{H^{\prime} A \rho^{2}\left(1-Z^{2}\right)}\right) \mathrm{d} \rho=0 .
$$

The first integral is divergent because $\lim _{\rho \rightarrow \rho_{1}} \ln H^{\prime}=\infty$. The second and third terms are integrable (remember that $H^{\prime 2}$ is integrable). Thus, to get a contradiction it suffices to show that the last term is integrable. We write this term as

$$
\frac{\sin (2 H)}{H^{\prime} A \rho^{2}\left(1-Z^{2}\right)}=\frac{\sin (2 H)}{H A \rho^{2}} \frac{H}{\left(1-Z^{2}\right) H^{\prime}} .
$$

The first factor is continuous and we now show that the second factor is also continuous. Applying L'Hôpital's rule, we get

$$
\lim _{\rho \rightarrow \rho_{1}} \frac{H}{\left(1-Z^{2}\right) H^{\prime}}=\lim _{\rho \rightarrow \rho_{1}} \frac{H^{\prime}}{-2 Z Z^{\prime} H^{\prime}+\left(1-Z^{2}\right) H^{\prime \prime}}=\lim _{\rho \rightarrow \rho_{1}} \frac{1}{-2 Z Z^{\prime}+\left(1-Z^{2}\right) H^{\prime \prime} / H^{\prime}} .
$$

Next, using (21) we get

$$
\left(1-Z^{2}\right) \frac{H^{\prime \prime}}{H^{\prime}}=-\frac{2\left(1-Z^{2}\right)}{\rho}+\alpha \rho\left(1+Z^{2}\right)\left(1-Z^{2}\right) H^{\prime 2}-\frac{\sin (2 H)}{A \rho^{2} H^{\prime}} .
$$

In the limit $\rho \rightarrow \rho_{1}$, the first term on the rhs of (31) obviously goes to zero, the second does by proposition 2 and the third does by the assumption that $H^{\prime} \rightarrow \infty$. Thus, limit (30) is finite and consequently so is (29). This contradicts (28) and thus concludes the proof of the 'if' part of lemma 4.

Corollary 5. A c-orbit which has $\bar{H}(c)=0$ is analytic on the whole interval $0 \leqslant \rho \leqslant \rho_{1}$.

Proof. The boundedness of $H^{\prime}(\rho)$ implies by $(21)$ that $H^{\prime \prime}>-2 H^{\prime} / \rho$ is bounded below (remember that $H(\rho)<0$ and $H^{\prime}(\rho)>0$ near $\left.\rho_{1}\right)$, hence $\lim _{\rho \rightarrow \rho_{1}} H^{\prime}(\rho)$ exists. Having that, it follows that $\sin (2 H) /\left(1-Z^{2}\right)$ has a finite limit (since $\lim Z^{\prime}=1 / \rho_{1} \neq 0$ ), and therefore the solution $(H, A, Z)$ is $C^{2}$ near $\rho_{1}$. By a routine contraction mapping argument, one can show that $C^{2}$ solutions are unique, hence a $c$-orbit must belong to the one-parameter family of analytic solutions from proposition 14 (see the appendix). 
Next, we describe the behaviour of $c$-orbits for small and large values of the shooting parameter $c$. We define a nodal number of a $c$-orbit $N(c)=$ number of zeros of the function $H(\rho)$ on the interval $0 \leqslant \rho<\rho_{1}$. We first show that $c$-orbits with small $c$ have no nodes.

Proposition 6. If $c$ is sufficiently small then $N(c)=0$.

Proof. For $c=0$ we have $H(\rho) \equiv-\pi / 2$ and $Z(\rho)=\rho$, so $\rho_{1}(c=0)=1$. By continuity, for any $\epsilon>0$ and sufficiently small $c$ we can find $\rho_{0}$ such that $1-\epsilon<\rho_{0}<\rho_{1}(c)<1$ and $H\left(\rho_{0}\right)<-\pi / 2+\epsilon$. We know from the proof of proposition 2 that $\lim _{\rho \rightarrow \rho_{1}} \sqrt{\rho_{1}-\rho} H^{\prime}=0$, hence

$$
H\left(\rho_{1}\right)-H\left(\rho_{0}\right)=\int_{\rho_{0}}^{\rho_{1}} H^{\prime}(\rho) \mathrm{d} \rho<\mathrm{const} \int_{\rho_{0}}^{\rho_{1}} \frac{\mathrm{d} \rho}{\sqrt{\rho_{1}-\rho}}<\text { const } \sqrt{\epsilon}
$$

Thus, $H(\rho)$ stays arbitrarily close to $-\pi / 2$ all the way up to $\rho_{1}$ if $c$ is sufficiently small and therefore $N(c)=0$. We remark that using a scaling argument one can derive the precise asymptotic behaviour of $c$-orbits for small $c$. We omit this argument since it is not needed for the proof.

We show next that $c$-orbits with large $c$ have arbitrarily many nodes.

Proposition 7. $N(c) \rightarrow \infty$ for $c \rightarrow \infty$.

Proof. We rescale the variables, setting

$x=c \rho, \quad \tilde{H}(x)=H(\rho), \quad \tilde{A}(x)=A(\rho), \quad \tilde{Z}(x)=c Z(\rho)$.

Then, equations (21)-(24) become

$$
\begin{aligned}
& \tilde{H}^{\prime \prime}+\frac{2}{x} \tilde{H}^{\prime}-\alpha\left(1+\frac{\tilde{Z}^{2}}{c^{2}}\right) x H^{\prime 3}+\frac{\sin (2 \tilde{H})}{\tilde{A} x^{2}\left(1-\frac{\tilde{Z}^{2}}{c^{2}}\right)}=0, \\
& \tilde{A}^{\prime}=-2 \alpha x \tilde{A} \tilde{H}^{\prime 2}, \\
& x \tilde{Z}^{\prime}=\tilde{Z}\left(1+\alpha\left(1-\frac{\tilde{Z}^{2}}{c^{2}}\right) x^{2} \tilde{H}^{\prime 2}\right),
\end{aligned}
$$

with the constraint

$$
1-2 \alpha-\tilde{A}+2 \alpha \sin ^{2} \tilde{H}+\alpha \tilde{A} x^{2} \tilde{H}^{\prime 2}\left(1-\frac{\tilde{Z}^{2}}{c^{2}}\right)=0,
$$

and the initial conditions at $x=0$

$$
\tilde{H}(0)=-\frac{\pi}{2}, \quad \tilde{H}^{\prime}(0)=1, \quad \tilde{A}(0)=1, \quad \tilde{Z}(0)=0, \quad \tilde{Z}^{\prime}(0)=1 .
$$

As $c \rightarrow \infty$, the solutions of equations (34)-(38) tend uniformly on compact intervals to solutions of the limiting equations

$$
\begin{aligned}
& h^{\prime \prime}+\frac{2}{x} h^{\prime}-\alpha x h^{\prime 3}+\frac{\sin (2 h)}{a x^{2}}=0, \\
& a^{\prime}=-2 \alpha x a h^{\prime 2}, \\
& x z^{\prime}=z\left(1+\alpha x^{2} h^{\prime 2}\right),
\end{aligned}
$$


with the constraint

$$
1-2 \alpha-a+2 \alpha \sin ^{2} h+\alpha a x^{2} h^{\prime 2}=0,
$$

and the same initial conditions at $x=0$,

$$
h(0)=-\frac{\pi}{2}, \quad h^{\prime}(0)=1, \quad a(0)=1, \quad z(0)=0, \quad z^{\prime}(0)=1 .
$$

We observe first that the function $a(x)$ is monotone decreasing by (40) and bounded below, $a>1-2 \alpha$, by (42). Thus, no singularity can develop due to $a$ going to zero. Also, by (42) no singularity can develop due to $h^{\prime}$ becoming unbounded. Thus, solutions exist for all $x>0$ (assuming the existence of a solution for small $x$ ). In order to complete the proof it is sufficient to show that the function $h(x)$ has an infinite number of zeros for $x>0$. Since $a<1$, it follows from (42) that $-\pi / 2<h(x)<\pi / 2$ for all $x>0$. To show that $h(x)$ oscillates around zero we consider three cases:

(i) Assume that $\lim _{x \rightarrow \infty} h(x)$ does not exist. Then, there must be a sequence $\cdots x_{k}<y_{k}<$ $x_{k+1}<y_{k+1}<\cdots$ such that $h$ has a local minimum at $x_{k}$ and a local maximum at $y_{k}$. By (39), $h^{\prime}\left(x_{k}\right)=0, h^{\prime \prime}\left(x_{k}\right) \geqslant 0$ imply that $\sin \left(2 h\left(x_{k}\right)\right) \leqslant 0$, hence $h\left(x_{k}\right) \leqslant 0$. By a similar argument, $h\left(y_{k}\right) \geqslant 0$. Thus, $h(x)$ has a zero in each interval $x_{k}<x<y_{k}$.

(ii) Assume that a nonzero $\lim _{x \rightarrow \infty} h(x)$ exists. Then, from (42) $\lim _{x \rightarrow \infty} x^{2} h^{\prime 2}$ exists and, in fact, equals zero because $\lim _{x \rightarrow \infty} h(x)$ exists. This implies by (39) that $\lim _{x \rightarrow \infty} x^{2} h^{\prime \prime}(x)=-\sin (2 h(\infty)) / A(\infty) \neq 0$, hence $\lim _{x \rightarrow \infty} x^{2} h^{\prime 2}(x) \neq 0$. Thus case (ii) does not arise.

(iii) Assume that $\lim _{x \rightarrow \infty} h(x)=0$. We define the rotation function $\theta(x)$ by

$$
\tan \theta(x)=\frac{x h^{\prime}(x)}{h(x)}, \quad \theta(0)=0
$$

Remark 1. The rotation function $\theta(x)$ is well defined because the case $h(x)=h^{\prime}(x)=0$ is impossible for solutions satisfying the initial conditions (43). To see this, assume that $h\left(x_{0}\right)=h^{\prime}\left(x_{0}\right)=0$ for some $x_{0}>0$. Then, by (42) $a\left(x_{0}\right)=1-2 \alpha$ and the unique solution with these initial conditions at $x_{0}$ is $h(x)=0, a(x)=1-2 \alpha$ for all $x$, contradicting the initial conditions (43).

We want to show that $\lim _{x \rightarrow \infty} \theta(x)=-\infty$. Using (39) we obtain

$$
x \theta^{\prime}(x)=-\sin ^{2} \theta-\frac{\sin 2 h}{2 h} \frac{2 \cos ^{2} \theta}{a}-\frac{\left(1-2 \alpha \cos ^{2} h\right) \sin \theta \cos \theta}{a} .
$$

Under the assumption $\lim _{x \rightarrow \infty} h(x)=0$, it follows from (42) that $\lim _{x \rightarrow \infty} a(x)=1-2 \alpha$, hence for sufficiently large $x$

$$
\theta^{\prime}(x) \approx-\frac{1}{x}\left(\sin ^{2} \theta+\sin \theta \cos \theta+\frac{2 \cos ^{2} \theta}{1-2 \alpha}\right)<-\frac{3}{4 x},
$$

so $\lim _{x \rightarrow \infty} \theta(x)=-\infty$. Thus, given any integer $k$ there exists an $x_{k}$ such that $h(x)$ has at least $k$ zeros for $x<x_{k}$. By continuous dependence on initial conditions, we may choose $c>x_{k} / \sqrt{1-2 \alpha}$ so that the $c$-solution has $k$ zeros also for $x<x_{k}$. In terms of the variable $\rho=x / c$ the $c$-solution has $k$ zeros for $\rho<\sqrt{1-2 \alpha}<\rho_{1}(c)$. This completes the proof of proposition 7 .

Next, we need two lemmas which tell us how the number of nodes $N(c)$ changes under small variations of $c$.

Lemma 8. If $\bar{H}(\tilde{c})=0$, then $N(c)=N(\tilde{c})$ or $N(c)=N(\tilde{c})+1$ for c sufficiently close to $\tilde{c}$. 
Proof. First note that if $H(\rho, \tilde{c})$ has a zero at some $\rho_{0}<\rho_{1}(\tilde{c})$, then $H^{\prime}\left(\rho_{0}, \tilde{c}\right) \neq 0$ (see remark 1), so by continuity of $H(\rho, c)$ with respect to $c, H(\rho, c)$ also has a zero if $c$ is sufficiently close to $\tilde{c}$. Thus $N(c) \geqslant N(\tilde{c})$ and it suffices to show that $N(c) \leqslant N(\tilde{c})+1$. Let $\tilde{a}<\rho_{1}(\tilde{c})$ be the last node of the $\tilde{c}$-orbit, that is $H(\tilde{a}, \tilde{c})=0$ and, for concreteness, $H(\rho, \tilde{c})<0$ for $\tilde{a}<\rho<\rho_{1}$. By continuity with respect to $c, H(\rho, c)$ will also have a zero at $a$ near $\tilde{a}$ if $c$ is near $\tilde{c}$. In order to prove that $H(\rho, c)$ cannot have more than one zero in the interval $a<\rho<\rho_{1}(c)$, we now show that if $H(\rho, c)$ becomes positive for some $\rho>a$, then it would not have time to change the sign again before going singular. Assume for contradiction that there is a segment $a<\rho_{N} \leqslant \rho \leqslant \rho_{D}$ of the $c$-orbit in which the function $H(\rho)$ is monotone decreasing from a local maximum $H\left(\rho_{N}\right)>0$ to $H\left(\rho_{D}\right)=0$.

We define

$$
W=\frac{1}{2} \rho^{2} A H^{\prime 2}\left(1-Z^{2}\right)+\sin ^{2} H .
$$

From (24) $W=(A-1+2 \alpha) /(2 \alpha)$, hence by $(22) W^{\prime}<0$. We have

$$
\frac{H^{\prime 2}}{W-\sin ^{2} H}=\frac{2}{\rho^{2} A\left(1-Z^{2}\right)}, \quad \text { so } \quad \frac{-H^{\prime}}{\sqrt{W-\sin ^{2} H}}=\frac{\sqrt{2}}{\rho \sqrt{A\left(1-Z^{2}\right)}} \text {. }
$$

Integrating the left-hand side from $\rho_{N}$ to $\rho_{D}$, we get (using $H_{N}=H\left(\rho_{N}\right)$ )

$$
\int_{\rho_{N}}^{\rho_{D}} \frac{-H^{\prime} \mathrm{d} \rho}{\sqrt{W-\sin ^{2} H}}=\int_{0}^{H_{N}} \frac{\mathrm{d} H}{\sqrt{W-\sin ^{2} H}} \geqslant \int_{0}^{H_{N}} \frac{\mathrm{d} H}{\sqrt{\sin ^{2} H_{N}-\sin ^{2} H}}>\frac{\pi}{2},
$$

where the first inequality follows from $W(\rho) \leqslant W\left(\rho_{N}\right)=\sin ^{2} H_{N}$ (since $W^{\prime}$ decreases) and the second inequality is a simple calculation using a substitution $\sin H=u \sin H_{N}$ (remember that $\left.H_{N}<\pi / 2\right)$.

Next, we derive an upper bound for the integral of the right-hand side of (48). We have

$\int_{\rho_{N}}^{\rho_{D}} \frac{\mathrm{d} \rho}{\rho \sqrt{A\left(1-Z^{2}\right)}} \leqslant \frac{1}{\rho_{N} \sqrt{1-2 \alpha}} \int_{\rho_{N}}^{\rho_{D}} \frac{\mathrm{d} \rho}{\sqrt{1-Z^{2}}} \leqslant \frac{1}{\rho_{N} \sqrt{1-2 \alpha}} \int_{\rho_{N}}^{\rho_{D}} \frac{\mathrm{d} \rho}{\sqrt{1-Z}}$.

We showed above that $Z^{\prime}>1$, hence $1-Z \geqslant \rho_{1}-\rho$. Therefore

$\int_{\rho_{N}}^{\rho_{D}} \frac{\mathrm{d} \rho}{\sqrt{1-Z}} \leqslant \int_{\rho_{N}}^{\rho_{D}} \frac{\mathrm{d} \rho}{\sqrt{\rho_{1}-\rho}}=2\left(\sqrt{\rho_{1}-\rho_{N}}-\sqrt{\rho_{1}-\rho_{D}}\right)<2 \sqrt{\rho_{1}-\rho_{N}}$.

By continuity of solutions with respect to $c$ and by corollary $3, \rho_{N}$ is arbitrarily close to $\rho_{1}(c)$ if $c$ is sufficiently close to $\tilde{c}$, hence it follows from (51) that the integral of the right-hand side of (48) is arbitrarily small. This is in contradiction with (49), hence $H(\rho, c)$ cannot have a second additional zero, which completes the proof of lemma 8.

Lemma 9. If $\bar{H}(\tilde{c}) \neq 0$, then $N(c)=N(\tilde{c})$ for c sufficiently close to $\tilde{c}$.

Proof. Without the loss of generality, we assume that $\bar{H}(\tilde{c})<0$. As above, let $\tilde{a}<\rho_{1}(\tilde{c})$ be the last node of the $\tilde{c}$-orbit, that is $H(\tilde{a}, \tilde{c})=0$ and $H(\rho, \tilde{c})<0$ for $\tilde{a}<\rho \leqslant \rho_{1}$. Let $a$ be the corresponding zero of $H(\rho, c)$ for $c$ near $\tilde{c}$. We want to show that $H(\rho, c)$ cannot have an extra zero for $\rho>a$. Suppose for contradiction that $H(b, c)=0$ for some $b>a$. For $\bar{H}(\tilde{c})<0$ we have $H^{\prime}(\rho, \tilde{c})>0$ near $\rho_{1}(\tilde{c})$, so for solutions with $c$ sufficiently close to $\tilde{c}$ there must be a $\delta<b$ such that $H(\delta, c)=\bar{H}(\tilde{c})$. Let us integrate the identity

$$
\frac{H^{\prime}}{\sqrt{W-\sin ^{2} H}}=\frac{\sqrt{2}}{\rho \sqrt{A\left(1-Z^{2}\right)}}
$$

from $\delta$ to $b$. For the left-hand side, we get

$$
\int_{\delta}^{b} \frac{H^{\prime} \mathrm{d} \rho}{\sqrt{W-\sin ^{2} H}}=\int_{0}^{-\bar{H}} \frac{\mathrm{d} H}{\sqrt{W-\sin ^{2} H}} .
$$


From proposition 2 we know that $\lim _{\rho \rightarrow \rho_{1}}\left(1-Z^{2}\right) H^{\prime 2}=0$, so $W(\rho, \tilde{c})<(1+\epsilon / 2) \sin ^{2} \bar{H}$ for $\rho$ near $\rho_{1}$ and hence $W(\rho, c)<(1+\epsilon) \sin ^{2} \bar{H}$ for $c$ near $\tilde{c}$. Since $W$ is decreasing, $W(\delta, c)<W(\rho, c)<(1+\epsilon) \sin ^{2} \bar{H}$. Thus

$\int_{0}^{-\bar{H}} \frac{\mathrm{d} H}{\sqrt{W-\sin ^{2} H}} \geqslant \int_{0}^{-\bar{H}} \frac{\mathrm{d} H}{\sqrt{(1+\epsilon) \sin ^{2} \bar{H}-\sin ^{2} H}} \geqslant \arcsin \left(\frac{1}{\sqrt{1+\epsilon}}\right)>\frac{\pi}{2}$

for sufficiently small $\epsilon$, where the last but one inequality can be seen by substituting $\sin H=u \sin \bar{H}$ into the integral. By the same argument as in the proof of lemma 8 , the integral of the right-hand side of $(52)$ is $O\left(\sqrt{\rho_{1}-\rho}\right)$. By continuity of solutions with respect to $c$ and by corollary $3, \delta$ is arbitrarily close to $\rho_{1}(c)$ if $c$ is sufficiently close to $\tilde{c}$, hence the integral of the left-hand side of equation (52) is arbitrarily small. This contradicts (54) and completes the proof of lemma 9.

Now we are ready to make a shooting argument. We define a set

$$
C_{0}=\{c \mid N(c)=0\}
$$

and let $c_{0}=\sup C_{0}$. The set $C_{0}$ is nonempty (by proposition 6) and bounded above (by proposition 7) so $c_{0}$ exists. We claim that the $c_{0}$-orbit has no nodes and satisfies the boundary condition $\bar{H}\left(c_{0}\right)=0$. To see this, note that the $c_{0}$-orbit cannot have a node because then by lemmas 8 and 9 all nearby $c$-orbits would have a node, so there would be an interval around $c_{0}$ without any elements of $C_{0}$ in it, contradicting the assumption that $c_{0}$ is the least upper bound. Thus, $N\left(c_{0}\right)=0$. Now, if $\bar{H}\left(c_{0}\right)<0$, then by lemma 9 all nearby $c$-orbits would have no nodes, so there would be an interval around $c_{0}$ consisting of elements of $C_{0}$, contradicting the assumption that $c_{0}$ is an upper bound of $C_{0}$. Thus $\bar{H}\left(c_{0}\right)=0$.

Next, we define $C_{1}=\left\{c>c_{0} \mid N(c)=1\right\}$. This set is nonempty by the previous step and lemma 8 and bounded above by proposition 7 , hence $c_{1}=\sup C_{1}$ exists. By the same argument as above, the $c_{1}$-orbit has exactly one node and satisfies $\bar{H}\left(c_{1}\right)=0$. The construction of subsequent $c_{n}$-orbits proceeds by induction.

\subsection{Conclusion of the proof of theorem 1}

Returning to the original variable $F(\rho)$ and rescaling $\rho \rightarrow \rho / \rho_{1}\left(c_{n}\right)$ we get the solution of equations (13)-(17) which satisfies the boundary conditions (19) and (20) and has exactly $n$ intersections with the line $F=\pi / 2$. By corollary 5 this solution is analytic in the whole interval $0 \leqslant \rho \leqslant 1$.

\section{Beyond the past self-similarity horizon}

In this section, we consider the behaviour of the CSS solutions of theorem 1 outside the past SSH; in particular, we ask the question: do these solutions possess a regular future selfsimilarity horizon? Note that $\rho=\infty$ corresponds to the hypersurface $(t=0, r>0)$ so in order to analyse the global behaviour of solutions (for $t>0$ ) we need to go 'beyond $\rho=\infty$ '. To this end, we define, after I, a new coordinate $x$ by

$$
\frac{\mathrm{d}}{\mathrm{d} x}=\rho Z \frac{\mathrm{d}}{\mathrm{d} \rho}, \quad x(\rho=1)=0 .
$$

We also define an auxiliary function $w(x)=1 / Z(\rho)$. In these new variables, the past SSH where $w=1$ is at $x=0$, while the future SSH (if it exists) is at some $x_{A}>0$ where $w\left(x_{A}\right)=-1$. 

$\mathrm{d} / \mathrm{d} x)$

In terms of $x$ and $w$, equations (21)-(23) become autonomous (where a prime is now

$$
\begin{aligned}
& H^{\prime \prime}-2 \alpha w H^{\prime 3}+\frac{\sin (2 H)}{A\left(w^{2}-1\right)}=0 \\
& A^{\prime}=-2 \alpha A w H^{\prime 2} \\
& w^{\prime}=-1+\alpha\left(1-w^{2}\right) H^{\prime 2}
\end{aligned}
$$

The constraint (24) becomes

$$
1-2 \alpha-A+2 \alpha \sin ^{2} H+\alpha A H^{\prime 2}\left(w^{2}-1\right)=0 .
$$

From (20) the initial conditions at $x=0$ are

$$
H(x) \sim b x, \quad w(x) \sim 1-x, \quad A(x) \sim 1-2 \alpha-2 \alpha(1-2 \alpha) b^{2} x .
$$

We know from theorem 1 that for each $\alpha<1 / 2$ there is an infinite sequence $\left\{b_{n}(\alpha)\right\}$ determining solutions which are regular inside the past SSH, that is, for all $x \leqslant 0$ (note that $\rho=0$ corresponds to $x=-\infty)$. In I we showed that for $x>0$ the solutions starting from the past SSH with the initial conditions (61) tend in finite 'time' to $w=-1$ if $b$ is small, or to $w=+1$ if $b$ is large. After I we shall refer to these two kinds of behaviour as type A and type B solutions, respectively. Now we want to show that the solutions of theorem 1 are of type A (and therefore possess the future SSH) provided that $\alpha$ is sufficiently small. Unfortunately, the shooting argument gives us insufficient information about the parameters $b_{n}$, so we cannot apply the above-mentioned result of I to determine the character of solutions outside the past $\mathrm{SSH}$. Instead, we shall make use of the obvious fact that for $\alpha=0$ all solutions are of type A.

Lemma 10. For sufficiently small $\alpha$ the $c_{n}$-orbits of theorem 1 (rescaled so that $\rho_{1}(c)=1$ ) have $\left|b_{n}\right|$ uniformly bounded above for all $n$.

Proof. It was shown in [2] (see lemma 4 in that reference) that for $\alpha=0$ the solution to equations (57)-(61) for $x<0$ must exit the strip $|H| \leqslant \pi / 2$ if $|b|$ is too large, say $|b|>B$. By continuous dependence, the same is true for sufficiently small $\alpha$. However, from proposition 2 the $c$-orbits must stay in the strip $|H| \leqslant \pi / 2$ for all $x<0$. Thus, $\left|b_{n}\right| \leqslant B$ for small $\alpha$.

Lemma 11. If a solution to equations (57)-(60) has $w\left(x_{0}\right)<0$ and $A\left(x_{0}\right)>1 / 2$ for some $x_{0}$, then there is $x_{A}>x_{0}$ such that $\lim _{x \rightarrow x_{A}} w(x)=-1$, i.e., the solution is of type $A$.

Proof. By (58) $A$ is increasing for $w<0$. Thus, using equation (59) and the constraint (60) we get for $x>x_{0}$

$w^{\prime}=-1+\alpha\left(1-w^{2}\right) H^{\prime 2}=-1+\frac{1-A-2 \alpha \cos ^{2} H}{A}<-2+\frac{1}{A\left(x_{0}\right)}<0$,

hence $w$ must hit -1 for some finite $x_{A}>x_{0}$.

Proposition 12. The $c_{n}(\alpha)$-orbits are of type $A$ if $\alpha$ is sufficiently small.

Proof. For $\alpha=0$ and any $b$ we have $w(x)=1-x$ and $A(x) \equiv 1$; in particular, $A(3 / 2)=1>1 / 2$ and $w(3 / 2)=-1 / 2<0$. By continuous dependence on initial conditions, there exists a $\delta(b)$ such that if $\alpha<\delta(b)$ and $\left|b-b^{\prime}\right|<\delta(b)$, then $A\left(3 / 2, b^{\prime}\right)>1 / 2$ and $w\left(3 / 2, b^{\prime}\right)<0$. This implies by lemma 11 that the solutions corresponding to such values of $\alpha$ and $b^{\prime}$ are of type A. By a standard theorem of advanced calculus, there is a $\delta^{\prime}>0$ (independent of $b$ ) such that the solutions with $\alpha<\delta^{\prime}$ and $|b| \leqslant B$ are of type A. By lemma 10 any $c_{n}$-orbit has $|b| \leqslant B$, so for $\alpha<\delta^{\prime}$ the $c_{n}$-orbits are of type A. 
By a similar argument as in the proof of proposition 2, one can easily show that the type A solutions are generically only $C^{0}$ at the future SSH (for isolated values of $\alpha$ there are solutions that go smoothly through the future SSH). In I we showed that the leading-order asymptotic behaviour at the future SSH is (using $y=x_{A}-x$ )

$w \sim-1+y, \quad A \sim A_{0}-2 \alpha A_{0} C^{2} y \ln ^{2}(y), \quad H \sim H_{0}-C y \ln (y)$,

where $A_{0}=1-2 \alpha \cos ^{2} H_{0}, C=\sin \left(2 H_{0}\right) / 2 A_{0}$ and $H_{0}$ is a free parameter. Using this expansion, one can check that the curvature is finite as $y \rightarrow 0$ which means that the type A solutions are examples of naked singularities.

\section{Acknowledgments}

We acknowledge the hospitality of the Erwin Schrödinger Institute for Mathematical Physics in Vienna, where part of this paper was produced. The research of PB was supported in part by the KBN grant 2 P03B 01016.

\section{Appendix (local existence theorems)}

In [5] (proposition 1) Breitelohner, Forgács and Maison derived the following result concerning the behaviour of solutions of a system of ordinary differential equations near a singular point (see also [6] for a similar result).

Theorem (BFM). Consider a system of first-order differential equations for $n+m$ functions $u=\left(u_{1}, \ldots, u_{n}\right)$ and $v=\left(v_{1}, \ldots, v_{m}\right)$,

$$
t \frac{\mathrm{d} u_{i}}{\mathrm{~d} t}=t^{\mu_{i}} f_{i}(t, u, v), \quad t \frac{\mathrm{d} v_{i}}{\mathrm{~d} t}=-\lambda_{i} v_{i}+t^{v_{i}} g_{i}(t, u, v),
$$

where constants $\lambda_{i}>0$ and integers $\mu_{i}, v_{i} \geqslant 1$ and let $C$ be an open subset of $R^{n}$ such that the functions $f$ and $g$ are analytic in the neighbourhood of $t=0, u=c, v=0$ for all $c \in C$. Then there exists an n-parameter family of solutions of the system (64) such that

$$
u_{i}(t)=c_{i}+O\left(t^{\mu_{i}}\right), \quad v_{i}(t)=O\left(t^{\nu_{i}}\right),
$$

where $u_{i}(t)$ and $v_{i}(t)$ are defined for all $c \in C,|t|<t_{0}(c)$ and are analytic in $t$ and $c$.

We shall use this theorem to prove existence of local solutions of equations (21)-(23) near the singular points $\rho=0$ and $\rho=1$.

Proposition 13. Equations (21)-(23) admit a two-parameter family of local solutions near $\rho=0$,

$$
\begin{aligned}
& H(\rho)=-\frac{\pi}{2}+c \rho+O\left(\rho^{3}\right), \\
& A(\rho)=1-\alpha c^{2} \rho^{2}+O\left(\rho^{4}\right), \\
& Z(\rho)=d \rho+O\left(\rho^{3}\right),
\end{aligned}
$$

which are analytic in $c, d$ and $\rho$.

Proof. Using the variables

$$
w_{1}=\frac{H+\pi / 2}{\rho}, \quad w_{2}=H^{\prime}, \quad w_{3}=\frac{1-A}{\rho^{2}}, \quad w_{4}=\frac{Z}{\rho}
$$


we rewrite equations (21)-(23) as the first-order system

$$
\begin{array}{ll}
\rho w_{1}^{\prime}=-w_{1}+w_{2}, & \rho w_{2}^{\prime}=2 w_{1}-2 w_{2}+\rho^{2} h_{1}, \\
\rho w_{3}^{\prime}=-2 w_{3}+2 \alpha w_{2}^{2}+\rho^{2} h_{2}, & \rho w_{4}^{\prime}=\rho^{2} h_{3},
\end{array}
$$

where the functions $h_{i}$ are analytic near $\rho=0$. Next, substituting

we put (70) into the form (64)

$$
\begin{array}{ll}
w_{1}=u_{1}-v_{1}, & w_{2}=u_{1}+2 v_{1}, \\
w_{3}=v_{2}+\alpha\left(u_{1}^{2}-2 v_{1}^{2}-8 u_{1} v_{1}\right), & w_{4}=u_{2}
\end{array}
$$

$$
\begin{array}{ll}
\rho u_{1}^{\prime}=\rho^{2} f_{1}, & \rho u_{2}^{\prime}=\rho^{2} f_{2}, \\
\rho v_{1}^{\prime}=-3 v_{1}+\rho^{2} g_{1}, & \rho v_{2}^{\prime}=-2 v_{2}+\rho^{2} g_{2},
\end{array}
$$

where the functions $f_{i}, g_{i}$ are analytic in an open neighbourhood of $\rho=0, u_{1}=c, u_{2}=d$, $v_{i}=0$ for any $c$ and $d$. Thus, according to the BFM theorem, there exists a two-parameter family of solutions such that

$$
\begin{array}{ll}
u_{1}=c+O\left(\rho^{2}\right), & u_{2}=d+O\left(\rho^{2}\right), \\
v_{1}=O\left(\rho^{2}\right), & v_{2}=O\left(\rho^{2}\right),
\end{array}
$$

which is equivalent to (66)-(68).

Proposition 14. Equations (21)-(23) admit a one-parameter family of local solutions near $\rho=1$,

$$
\begin{aligned}
& H(\rho)=b(\rho-1)+O\left((\rho-1)^{2}\right) \\
& A(\rho)=1-2 \alpha-2 \alpha(1-2 \alpha) b^{2}(\rho-1)+O\left((\rho-1)^{2}\right) \\
& Z(\rho)=\rho+O\left((\rho-1)^{2}\right)
\end{aligned}
$$

which are analytic in $b$ and $\rho$.

Proof. We define the variables

$$
\begin{aligned}
& u=H^{\prime}, \quad v_{1}=\frac{H}{\rho-1}-H^{\prime}, \\
& v_{2}=\frac{(1-2 \alpha)-A}{\rho-1}-2 \alpha(1-2 \alpha) H^{\prime 2}, \quad v_{3}=\frac{Z-1}{\rho-1}-1 .
\end{aligned}
$$

Then, equations (21)-(23) take the form (using $t=\rho-1)$

$$
t u^{\prime}=t f, \quad t v_{i}^{\prime}=-v_{i}+t g_{i},
$$

where the functions $f$ and $g_{i}$ are analytic in an open neighourhood of $t=0, u=b, v_{i}=0$ for any $b>0$. Thus, according to the BFM theorem, there exists a one-parameter family of solutions such that

$$
u(t)=b+O(t), \quad v_{i}(t)=O(t),
$$

which is equivalent to (75)-(77).

\section{References}

[1] Bizoń P and Wasserman A 2000 Self-similar spherically symmetric wave maps coupled to gravity Phys. Rev. D 62084031

[2] Bizoń P 2000 Equivariant self-similar wave maps from Minkowski spacetime into 3-sphere Commun. Math Phys. 21545 
[3] Lechner C, Thornburg J, Husa S and Aichelburg P C 2001 A new transition between discrete and continuous self-similarity in critical gravitational collapse Preprint gr-qc/0112008

[4] Lechner C 2001 PhD Thesis University of Vienna

[5] Breitenlohner P, Forgács P and Maison D 1994 On static spherically symmetric solutions of the Einstein-YangMills equations Commun. Math. Phys. 163141

[6] Rendall A D and Schmidt B G 1991 Existence and properties of spherically symmetric static fluid bodies with a given equation of state Class. Quantum Grav. 8985 